\title{
The Role of the Oral Healthcare Team in Identification of Type 2 Diabetes Mellitus: A Systematic Review
}

\author{
Zehra Yonel $^{1} \cdot$ Joanna Batt $^{1} \cdot$ Rosemarie Jane $^{2} \cdot$ Enzo Cerullo $^{3} \cdot$ Laura J Gray $^{3} \cdot$ Thomas Dietrich $^{1} \cdot$ lain Chapple $^{1,4}$ (D)
}

Published online: 18 January 2020

(C) The Author(s) 2020

\begin{abstract}
Purpose of Review Type 2 diabetes (T2DM) presents a growing global health and economic burden. Dental settings have been employed to identify individuals who may be at high risk of diabetes, who exhibit non-diabetic hyperglycaemia (NDH - also termed "prediabetes") and who already unknowingly have the condition, through the use of targeted risk-assessments. This review aims to synthesize the existing literature supporting dental teams' identification of individuals at an increased risk of or suffering from undiagnosed NDH or T2DM in dental specialist care settings.

Recent Findings Electronic databases were searched for studies reporting the identification of NDH and or T2DM, in specialist care dental settings. Screening of returned articles and data extraction were completed by two independent reviewers (RJ, ZY). A descriptive synthesis of the included articles was undertaken. Due to heterogeneity of the literature, a meta-analysis could not be performed. The search yielded 52 eligible studies, of which 12 focused primarily on stakeholder opinions. Opinions of patients, dentists, dental hygienists, dental students and physicians on case identification of T2DM by oral health professionals were generally positive. The main barriers cited were time, cost, inadequate training and low follow-up of participants by primary care physicians. The risk assessment processes varied, with most studies using a combination of methods consisting of a questionnaire followed by a chairside blood sample. Methods utilizing questionnaires, gingival crevicular blood (GCB), fingerstick blood (FSB) and urine samples have all been evaluated.

Summary This review demonstrates that there may be benefit in engaging the dental workforce to identify cases of NDH and undiagnosed T2DM and that such a care pathway has the support of multiple stakeholders. Further high-quality research is required to assess both the clinical and cost-effectiveness of such practice in order to optimize protocols and patient care pathways. Studies should include a comparison of methods, health economic analyses and protocols to ensure those identified as high-risk go on to receive appropriate follow-up care.
\end{abstract}

Keywords Type 2 diabetes $\cdot$ Non-diabetic hyperglycaemia $\cdot$ Screening $\cdot$ Risk assessment $\cdot$ Dental settings

This article is part of the Topical Collection on Host Parasite Interactions in Periodontal Disease

Electronic supplementary material The online version of this article (https://doi.org/10.1007/s40496-020-00250-w) contains supplementary material, which is available to authorized users.

Iain Chapple

I.L.C.CHAPPLE@bham.ac.uk

1 Periodontology Research Group, School of Dentistry, Birmingham Dental Hospital, University of Birmingham, Birmingham, UK

2 The Eastman Dental Hospital, London, UK

3 Department of Health Sciences, University of Leicester, Leicester, UK

4 Institute of Clinical Sciences, College of Medical \& Dental Sciences, The University of Birmingham, Birmingham, UK

\section{Introduction}

Type 2 diabetes mellitus (T2DM) is a growing public health concern, affecting approximately 60 million people in Europe, which equates to $10 \%$ of those aged over 25 years. More than 422 million adults are living with the condition globally according to the World Health Organization [1]. In 2017, 1 in 2 people (212 million individuals in total) were living with undiagnosed T2DM [2]. Additionally, many individuals with T2DM may remain undiagnosed for many years due to T2DM being asymptomatic in its early stages. This has implications for the secondary prevention and management of the condition [2]. Therefore, there is merit in exploring non- 
traditional approaches to enhance early identification of individuals with non-diabetic hyperglycaemia (NDH) and undiagnosed T2DM.

$\mathrm{NDH}$ or impaired glucose regulation refers to elevated blood glucose levels that are not yet in the diabetes range. In addition to an increased risk of T2DM, individuals with NDH are also at increased risk of developing cardiovascular conditions [2]. By identifying NDH early, it can aid in the primary prevention of T2DM. The International Diabetes Federation reported that in 2017 over 325 million people were at high risk of developing T2DM. These people are classified as having NDH or prediabetes [3].

The Global Burden of Diseases, Injuries, and Risk Factors Study 2017 (GBD 2017) reported that from 1990 to 2017, oral diseases (mainly periodontitis and caries) contributed the most years lost due to disability (YLD) in age-standardized prevalence rates from 354 diseases and injuries across 195 countries [4•]. Severe periodontitis affects $11.2 \%$ of adults worldwide [5]. Milder forms are even more prevalent, affecting $50 \%$ of adults and $60 \%$ of individuals over the age of 65 . Importantly, severe periodontitis is significantly and independently associated with T2DM [6•, 7-9]. Furthermore, glycaemic status directly impacts oral health [10]. Poor glycaemic control brings unwelcome consequences for periodontal health which ultimately, if left untreated, may lead to tooth loss and associated psychosocial sequelae [6•, 11-18]. Due to the well-established bidirectional relationship between periodontitis and T2DM, improvements in periodontal status can lead to improvements in diabetes control [6•, 9, 18-23], as evidenced by a recent randomized control trial which demonstrated a $0.6 \%$ reduction in $\mathrm{HbA} 1 \mathrm{c}$ at 12 months among patients who had received intensive periodontal therapy [24]. Screening for periodontitis is an established and mandatory procedure within dental settings in many countries around the world, providing information on oral risk factors for diabetes that primary care physicians are unable to assess.

In addition to the human cost of diabetes and periodontitis, and associated morbidity and mortality, there is a significant economic burden associated with both diseases. Severe periodontitis is estimated to cost $\$ 54$ billion (US dollars or USD) per year globally in lost productivity [25]. Periodontitis is also a major contributor to the aggregate direct treatment costs of oral disease, estimated at $\$ 91.05$ billion (USD) for western Europe and \$297.67 billion (USD) worldwide in 2010 . These were considerably higher when aggregated with indirect costs, amounting to $\$ 442$ billion (USD) [26].

It is in the interests of dental teams to know whether their patients have NDH or undiagnosed T2DM, due to the impact of both upon periodontal stability and treatment outcomes. Given the inter-relationship between these two chronic, noncommunicable diseases, raising awareness of the NDH/ T2DM status of patients in the dental environment will enable dental teams to better target their prevention and management strategies to improve oral health. Moreover, earlier detection of both conditions will facilitate improved systemic health outcomes for these individuals by facilitating appropriate prevention and interventions, further demonstrating the role that dental teams can play in assisting with management of the growing health and economic burden of T2DM.

\section{Aims and Objectives}

The objective of this review is to synthesize current evidence supporting dental teams' identification of individuals at an increased risk of or suffering from undiagnosed NDH or T2DM in dental specialist care settings. Evidence evaluated includes the opinions of key stakeholders, barriers to and facilitators of subject identification and the clinical methods used.

\section{Materials and Methods}

The present study was undertaken using a pre-specified protocol and reported according to the "Preferred Reporting Items for Systematic Reviews and Meta-Analyses” (PRISMA) guidelines for conducting and reporting systematic reviews and meta-analyses [27].

The Population, Intervention, Reference standard, Target condition (PIRT) format was followed for this review whereby $[\mathrm{P}]$ were stakeholders in the delivery of dental care, including patients aged greater than 18 years attending dental services, healthcare professionals and organizations involved in the delivery of dental care, [I] were the described screening modalities, [R] was the method by which NDH or T2DM was diagnosed and [T] was NDH or T2DM.

\section{Inclusion Criteria}

The inclusion criteria for the study included articles which met the following criteria:

- $\quad$ Risk assessment for NDH/ T2DM was undertaken in a hospital or specialist care dental setting.

- Opinions of stakeholders relating to diabetes risk assessment in dental settings were sought.

- Study subjects were adults ( $>18$ years of age).

- $\quad$ The article was written in the English language.

\section{Search Strategy}

Electronic bibliographic databases were searched, including MEDLINE, PubMed, the Cochrane Library, Clinicaltrials. gov and Web of Science. The reference lists of all eligible 
full texts were searched for additional papers. The search strategy (Table 1) included terms relating to or describing the identification of NDH or T2DM in dental settings. The search terms were adapted for use with other bibliographic databases. Restrictions to English language were applied and searches were limited to dates between January 1950 and October 2019.

\section{Data Extraction and Management}

The titles and abstracts of all returned papers were screened for the inclusion criteria. For included papers, full texts were reviewed, and any further exclusions determined by consensus. Reasons for exclusion at the full-text stage were recorded (Fig. 1). Electronic data extraction forms were developed, piloted and employed for all data extraction.

\section{Strategy for Synthesis}

It was anticipated that included studies would be highly heterogenous, resulting in a descriptive analytical approach. The descriptive synthesis was structured around the objectives of this review.

\section{Risk of Bias Assessment}

Two independent reviewers (ZY and JB) assessed the articles describing screening undertaken in a dental setting. Quality of the papers was assessed using a published and validated risk of bias assessment tool, the "United States Preventative Task Force Criteria for Assessing Internal Validity of Individual Studies".

Table 1 Search strategy: example of search strategy used in PubMed

\begin{tabular}{l} 
Search terms used \\
\hline (Screening[Title/Abstract] OR “risk \\
assessment”[Title/Abstract] OR “case \\
detection"[Title/Abstract] OR "case \\
finding”[Title/Abstract] OR “case \\
identification"[Title/Abstract] OR "risk \\
detection"[Title/Abstract] OR \\
diagnosis[Title/Abstract])) AND \\
(diabetes[Title/Abstract] OR \\
TTDM[Title/Abstract] OR T2DM \\
[Title/Abstract] OR \\
diabetic[Title/Abstract] OR \\
pre-diabetes[Title/Abstract] OR \\
prediabetes[Title/Abstract] OR \\
NDH[Title/Abstract] OR \\
hyperglycaemia[Title/Abstract] OR \\
hyperglycemia[Title/Abstract] OR \\
dysglycaemia[Title/Abstract] OR \\
dysglycemia[Title/Abstract])) AND \\
(dental[Title/Abstract] OR \\
dentistry[Title/Abstract] OR \\
dentist[Title/Abstract])
\end{tabular}

\section{Results}

The search strategy yielded 52 papers to be included. Eleven studies were focused on stakeholder opinion. Twenty-eight studies were primarily focused on undertaking risk assessments. The remaining studies had multiple elements including risk assessment and recording of stakeholder opinions.

The risk of bias assessment (Supplementary Table 2) demonstrated acceptable concordance between the two independent examiners (ZY and JB) with a kappa of 0.75 . Where there was disagreement in initial quality grade $(n=9)$, this was resolved through discussion and consensus in each case. The majority of articles were deemed to be of good quality, demonstrating a low risk of bias $(n=18)$. Seventeen articles showed a moderate risk of bias and one article was deemed to have a high risk of bias.

Eleven of the returned articles primarily focused on the opinions, attitudes and perceptions of stakeholders. Of these, five articles were related to patient perception, two to dental provider perceptions and the remaining four to dental hygienists, dental students, physicians and "authorities and organizations" (Table 2).

Among studies that asked patients whether they felt it was "important" that dentists identify individuals at high risk of T2DM, patient support was strong, in a range of $73 \%-87 \%$ [33, 35, 36, 39-41]. In addition to this acknowledgement, most patients surveyed were willing to undergo chairside screening methods that yielded immediate results and discuss the results of such tests with their dentist [35, 36, 39, 40].

In support of the positive patient opinion, one study reported that more than $60 \%$ of dentists surveyed believed that addressing T2DM was important to their role as a dentist, while $86 \%$ claimed to advise their patients with T2DM about their increased periodontal risk and $18 \%$ reported that they provided additional diabetes-related services [29]. Two-thirds of dentists in another study stated they would be interested in performing blood glucose monitoring if the costs were reimbursed [31]. A further study assessing the views of dentists reported that most felt it was important to conduct screening for diabetes $(76 \%)$, and $96 \%$ of respondents were willing to refer patients to a primary care physician for consultation. When methods of risk assessment were discussed, the majority were happy to collect oral fluids for salivary diagnostics $(88 \%)$ or conduct medical screening that yielded immediate results $(83 \%)$. The respondents in this particular study were significantly more willing to collect saliva than record height and weight measurements or undertake FSB collection. "Insurance" was also significantly less important to the dentists compared to time, cost, liability or patients' willingness [29].

In addition to the qualified dental workforce, one study sought the opinion of dental students to determine their opinions and willingness to assess patients for NDH/ T2DM. 
Fig. 1 PRISMA flow diagram for filtration of the search results

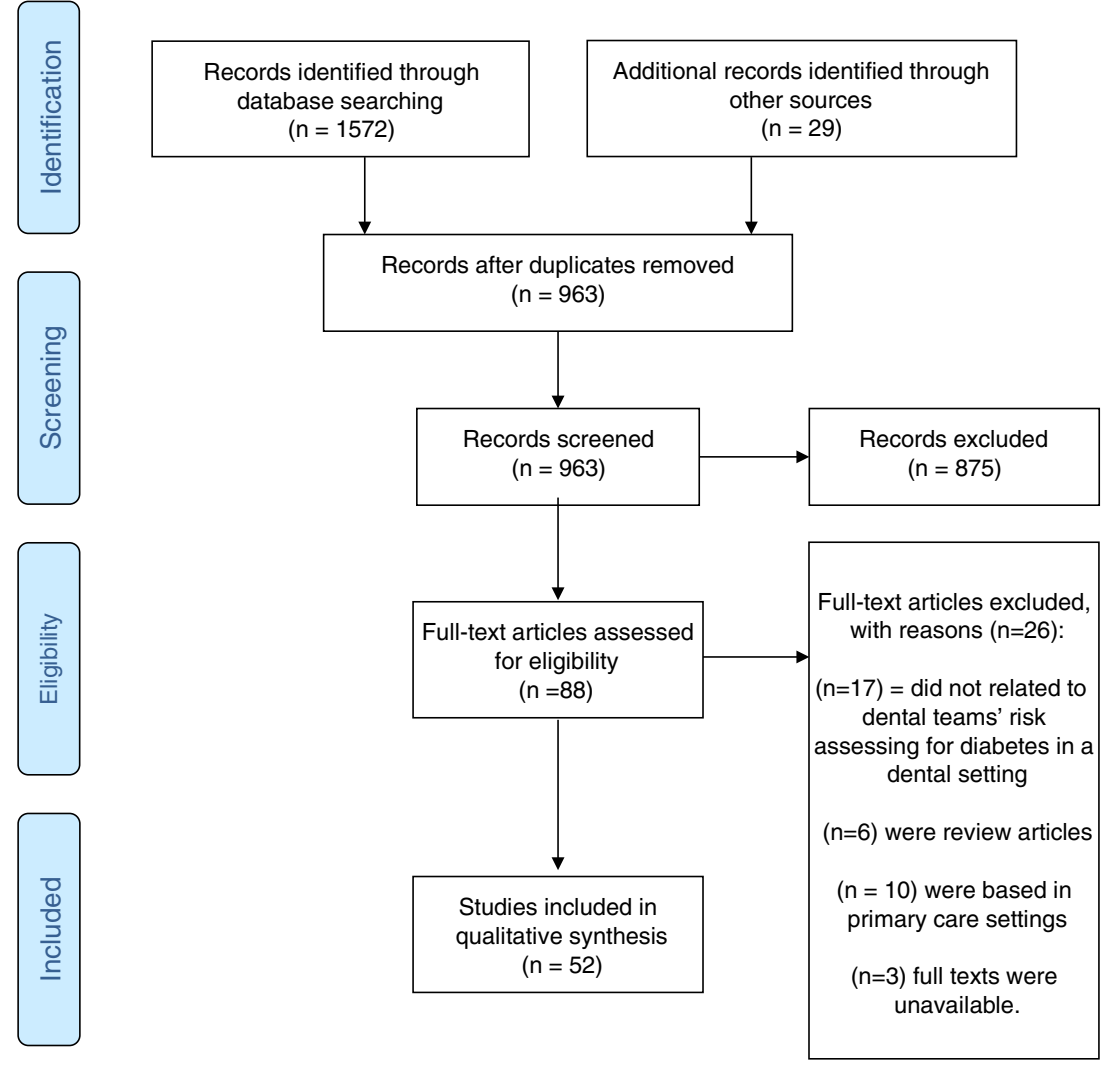

The principal barriers to undertaking screening for T2DM in dental settings were time, adequate training, support of the dentist or dental practice owner and patient willingness. Interestingly, in one study that assessed the willingness of racial/ethnic minority older adults to receive hypertension and diabetes screening as part of routine dental visits, five key themes emerged. These included that they found chairside risk assessment to be acceptable, that as older adults they found screening for conditions by healthcare professionals to be routine practice, that the interrelationship between oral and general health was appreciated, and that they perceived benefit to chairside screening. In some cases, it was also felt that chairside screening for general health conditions may reduce dental anxiety [35]. This study also identified key themes relating to patient-perceived barriers, which included that for some, dental fear may limit the acceptability of dental teams conducting chairside screening. Additional themes identified included that given the routine nature of screening for this demographic that there was a perceived lack of need for dental care and chairside screening in addition to a mistrust of dental providers as primary care providers among some of the sample [35].

Another concern cited was the poor follow-up rates with primary care medical practitioners. Poor follow-up was attributed to barriers including patients' inadequate knowledge about diabetes, lack of understanding about the importance of follow-up, "general business", financial concerns, fear 
Table 2 Summary of journal articles exploring stakeholder perceptions, attitudes and opinions to screening for diabetes in dental settings

\begin{tabular}{l} 
Paper \\
\hline $\begin{array}{l}\text { Anders et al. "Dental students' glucometer } \\
\text { experience and attitudes toward diabetes } \\
\text { counselling, monitoring, and screening: a } \\
\text { comparative study." }\end{array}$ \\
Esmeili et al. "Dentists' attitudes and \\
practices related to diabetes in the dental \\
setting."
\end{tabular}

[28] 2014 USA Dental students
setting."

Friman et al. "Medical screening in dental settings: a qualitative study of the views of authorities and organizations."

Greenberg et al. "Dentists' attitudes toward chairside screening for medical conditions."

Greenberg et al. "American dental hygienists' attitudes toward chairside medical screening in a dental setting."

[32] 2016 USA

Dental hygienists

2009 USA

Dentists

[30]

2015 Sweden $\begin{gathered}\text { Authorities \& } \\ \text { organization }\end{gathered}$

[31]

2010 USA

Dentists

(

Greenberg et al. "Patients' attitudes toward
screening for medical conditions in a

[33]

2012 USA Patients
Key finding (Barriers and facilitators) key-findings

Dental students' attitudes toward T2DM counselling, monitoring and screening were generally positive and more positive for those students who had greater experience using a glucometer. A high acceptance rate (84\%) for FSB among dental personnel who had hands-on experience using a glucometer was reported.

Sixty-one percent of respondents believed that addressing T2DM was important to their role, $86 \%$ advised patients with T2DM about periodontal risks, $18 \%$ provided T2DM-related services, $47 \%$ reported they knew how to assess for diabetes, and $42 \%$ felt well prepared to intervene with patients with diabetes. $66 \%$ of dentists reported interest in performing blood glucose monitoring if it was reimbursed.

Approached authorities and organizations generally had a positive view of medical screening in dental settings but were uncertain about the concept. Further scientific knowledge and guidelines concerning the topic are needed before it can be commonly introduced, alongside additional research on implementation strategies and long-term follow-up.

The majority thought it was important for dentists to conduct screening for T2DM (76.6\%). Respondents were willing to refer patients for consultation with physicians (96.4\%), collect oral fluids for salivary diagnostics $(87.7 \%)$, conduct medical screenings that yield immediate results $(83.4 \%)$ and collect blood via FSB $(55.9 \%)$. Respondents were significantly more willing $(P<.001)$ to collect saliva than height and weight measurements or FSB. Insurance was significantly less important $(P<.001)$ than time, cost, liability or patients' willingness."

Given that dental hygienists are involved in preventive and educational activities, medical screening seems like a natural extension to their roles. The majority of respondents (89\%) felt it was important to perform chairside screening for T2DM. Majorities were also willing to refer a patient for medical consult (94\%), conduct screening that yields immediate results $(85 \%)$ and to collect data/samples needed (57\%-95\%). The most important considerations were dentist/owner support (98\%), training (97\%), patient willingness (98\%) and time (98\%).

The majority of respondents were willing to have a dentist conduct screening for medical conditions with $73 \%$ specifically open to T2DM screening. The majority of clinic and private practice respondents were willing to let the dentist conduct screening that yields immediate results ( $90 \%$ vs $76 \%$ ), discuss results during their dental visit ( $89 \%$ vs $79 \%)$, refer them for a medical consult ( $86 \%$ vs $76 \%$ ) and send samples to an outside laboratory (76\% vs 59\%). Among potential barriers specified, both clinic and private practice respondents felt confidentiality 
Table 2 (continued)

Paper Reference Year Country Stakeholder Key finding (Barriers and facilitators) key-findings

Greenberg et al. "Physicians' attitudes toward medical screening in a dental setting."

Greenblatt et al. "Acceptability of Chairside Screening for Racial/Ethnic Minority Older Adults: A Qualitative Study.”
[34] 2015 USA Physician

[35]

2017 USA Patients

Sansare et al. "Indian patients' attitudes toward chairside screening in a dental setting for medical conditions."

Scambler et al. "Summary of: Patients' attitudes toward screening for diabetes and other medical conditions in the dental setting" was important ( $94 \%$ vs $83 \%$ ) followed by time (90\% vs $80 \%$ ) and insurance coverage ( $82 \%$ vs $80 \%$ ). Seventy-six percent of clinic respondents were willing to pay $\$ 10-20$, and $65 \%$ were willing to pay $\$ 21-30$; the percentage who were willing to pay more than $\$ 30$ dropped dramatically.

Of 1508 respondents, the majority felt it was valuable for dentists to conduct screening for T2DM (71\%). Respondents were willing to discuss results with the dentist (76\%) and accept patient referrals (89\%), and a small majority felt it was unimportant that the medical referral came from a dentist rather than a physician (52\%). The most important consideration was patient willingness (mean rank 2.55). Primary care physicians considered chairside medical screening in a dental setting to be valuable and worthwhile.

Five themes were manifest in the data regarding the willingness of racial/ethnic minority older adults to receive hypertension and T2DM screening as part of routine dental visits: (1) chairside screening is acceptable, (2) screening is routine for older adults, (3) the interrelationship between oral and general health is appreciated, (4) chairside screening has perceived benefits, and (5) chairside screening may reduce dental anxiety.Reservations centred on four major themes: (1) dental fear may limit the acceptability of chairside screening, (2) there is a perceived lack of need for dental care and chairside screening, (3) screening is available elsewhere, and (4) mistrust of dental providers as primary care providers.

A survey given to a convenience sample of adult patients visiting five university-based dental clinics (clinic group) and one private practice showed that both patient groups felt it was important for dentists to identify increased risk for medical conditions. The majority of patients were willing to have a dentist screen for specified conditions including T2DM (84.5\% clinic and $77.5 \%$ private). The majority of patients were willing to participate in chairside screening that yielded immediate results and discuss results immediately.

A self-administered questionnaire distributed to adult patients ( $\geq 18$ years) attending 2 primary care dental clinics and 16 general dental practices in South West England. Overall, 87\% of respondents thought that it was important or very important that dentists screened patients for medical conditions such as T2DM; $79 \%$ were very willing to let a dental team member carry out screening. Significantly higher proportions of respondents in the primary care clinics indicated willingness compared to general practices. Nearly two-thirds of primary care clinic respondents and over half of general practice patients indicated that they would be 
Table 2 (continued)

\begin{tabular}{|c|c|c|c|c|c|}
\hline Paper & Reference & Year & Country & Stakeholder & Key finding (Barriers and facilitators) key-findings \\
\hline & & & & & $\begin{array}{l}\text { willing to discuss test results with the dental } \\
\text { team. Overall, } 61 \% \text { had never knowingly been } \\
\text { screened or tested for T2DM; } 20 \% \text { reported that } \\
\text { they had been tested within the previous } \\
12 \text { months. }\end{array}$ \\
\hline $\begin{array}{l}\text { Rosedale et al. "Diabetes screening at the } \\
\text { periodontal visit: Patient and provider } \\
\text { experiences with two screening } \\
\text { approaches" }\end{array}$ & [38] & 2012 & USA & Patients and provider & $\begin{array}{l}\text { FSB samples from } 120 \text { patients and GCB samples } \\
\text { from } 102 \text { of these patients were collected on } \\
\text { special blood collection cards and sent to a } \\
\text { laboratory for HbA1c testing, with test results } \\
\text { sent to the patients from the laboratory. } \\
\text { Quantitative and qualitative data collection and } \\
\text { analyses of patients and providers were } \\
\text { conducted. Themes that arose from the } \\
\text { interviews with providers and patients include "a } \\
\text { good chance to check", "patient choice", "FSB } \\
\text { versus GCB testing" and "a new way of } \\
\text { interacting and viewing the dental visit". } \\
\text { Periodontal patients and dental providers believe } \\
\text { that the dental visit is an opportune site for } \\
\text { T2DM and generally prefer GCB to FSB } \\
\text { collection. GCB testing is well-tolerated, } \\
\text { convenient and acceptable to patients and } \\
\text { reduces time and liability obstacles for dental } \\
\text { providers to conduct T2DM screening. }\end{array}$ \\
\hline
\end{tabular}

and denial [38]. Only 53\% of participants with elevated $\mathrm{HbA} 1 \mathrm{c}$ values contacted their primary medical healthcare provider within 2 weeks as recommended in a pilot study [43]. Though a UK-based team also found that following up with a primary medical care provider was a potential barrier, they reported that patients were three times as likely to contact their general practitioner (GP) if they had received two positive screening results when compared with a patient with only one positive result [44]. A Swedish study with a 3-year follow-up of patients found that $89 \%$ had attended their healthcare provider within that time frame and that $9 \%$ of those had been formally diagnosed with T2DM. The study also identified that of those who had screened negative, $80.5 \%$ attended the primary healthcare centre, and eight $(0.6 \%)$ were found to have T2DM. Screening sensitivity was $52.9 \%$, and specificity was $90.6 \%$ with positive predictive value of $5.8 \%$. According to this study, when the population is limited to those $40-75$-year-olds with a BMI $>25 \mathrm{~kg} / \mathrm{m} 2$ and 30-to 75-year-olds with a BMI $>30 \mathrm{~kg} / \mathrm{m} 2$, the number needed to screen was 96 [45].

Twenty-eight articles returned from the search involved dental teams undertaking various screening methods within a specialist dental care setting (Supplementary Table 1). The majority of these studies $(n=10)$ employed a combination of screening methods, most commonly questionnaire followed by FSB sample collection. Within the literature, a range of screening methods were explored including questionnaires alone $(n=1)$, questionnaire in addition to another test modality $(n=6), \mathrm{FSB}$ collection alone $(n=4), \mathrm{FSB}$ in addition to another risk assessment modality $(n=11)$ and GCB collection $(n=6)$. Where blood samples were recorded, this was $\mathrm{HbA1c}$ in ten cases (Supplementary Table 1).

\section{Discussion}

\section{Principle Findings}

\section{Opinions of Key Stakeholders}

Overall the opinions of stakeholders relating to dental teams' engagement in risk assessment of patients for NDH/T2DM were positive. Furthermore, among patients there was strong support for tests which were able to yield immediate results. These findings appeared to transcend specific healthcare systems and cultural barriers, as similar results were reported irrespective of their being conducted in different countries with different models of healthcare provision, including state-funded healthcare systems in Europe [39-41], insurance-based and private healthcare within the USA [33] and university-based clinics and private settings in Asia [36].

Among methodologies currently in use, there was a general preference for GCB instead of FSB collection. GCB testing was well-tolerated among volunteers and was deemed both convenient and acceptable to patients. Additionally, it was 
found to reduce the obstacles of both time and liability for dental providers conducting the diabetes screening [38].

The sentiment expressed by patients, that dental teams undertaking risk-assessment for NDH/ T2DM was important, appeared to be shared by dental service providers [29, 31]. Dental hygienists also play a key role in the delivery of preventative advice and educational activities in dental practice. Thus, utilizing this skilled workforce in the delivery of NDH/ T2DM risk assessment appears to be a natural extension of their current duties, especially given their own strong support for such an intervention. Another key workforce to consider is primary care medical practitioners. Should any such additional services commence within dental settings, it is of paramount importance that any duplication of testing and generation of unnecessary referrals to primary care physicians is minimized. However, where physician opinion was sought in the literature, they were in support of the concept of utilizing dental teams to identify NDH /T2DM.

Given that patients, physicians and dental teams appear to be in favour of developing the role of the dental team to include risk-assessment for NDH/ T2DM, it was interesting to also understand the role of dental undergraduate students. These students are key to the future delivery of this additional service. One study based in the USA sought the attitudes of students toward counselling, monitoring and screening for T2DM; results were positive provided appropriate training in the required techniques was provided [28].

\section{Barriers and Facilitators}

Several patient-reported barriers and facilitators were identified within the literature including how the individuals' perception of dental teams and their own fears and anxiety may prevent uptake of additional services in such a setting. However, the patients also reported an appreciation of the interrelationship between oral and systemic disease and recognized potential benefit of routine testing by healthcare professionals [35].

A further barrier to dental teams undertaking risk assessment for NDH/ T2DM was the poor rate of follow-up with primary care medical practitioners for appropriate diagnosis and management. One study reported that while a majority of patients were interested in T2DM testing in dental offices, most dentists thought the tests were appropriate and simple to undertake and that T2DM screening in dental practice was deemed feasible; poor follow-up by patients, particularly those tested in private practices was a potential concern requiring further study [46].

In the UK based study that reported improved follow-up rates where two screening tests were undertaken, the screening was actually done by a researcher, and there was no analysis of the practices' additional workloads or their impact. Furthermore, the participants were contacted twice by telephone after their visit by the researcher and asked whether they had made an appointment with their GP. In many practices, there may be insufficient staff to undertake such additional steps, which may impact the outcome and result in an even further reduced rate of follow-up [44].

The most frequently cited barriers to uptake of new services were time and cost. Interestingly, a study in the USA found that the direct cost for each $\mathrm{HbAlc}$ test was \$9 (USD), excluding follow-up medical diagnosis. The mean screening time including patient education was reported as $14 \pm 6.2 \mathrm{~min}$. However, given the heterogeneity in methods used and devices available for undertaking each risk assessment method, the costs and time taken for risk assessment are likely to vary significantly. Interestingly, one study reported that $76 \%$ of patients were willing to pay $\$ 10-20$ (USD) and $65 \%$ were willing to pay \$21-30(USD). However, the percentage of who were willing to pay more than $\$ 30$ (USD) reduced dramatically [33].

\section{Risk Assessment Methods Used}

This systematic review revealed considerable variation in the methodologies adopted for risk assessing patients in dental settings for NDH/T2DM. Methods reported in the literature ranged from applying clinical guidelines and validated riskassessment tools to undertaking chairside testing of either GCB or FSB; urinalysis was also undertaken in some cases.

There are several methodologies accepted for use at each stage of study design. Screening criteria employed by studies analysed here were predominantly derived from the recommendations of the American Diabetes Association (ADA) or the National Institute for Clinical Excellence (NICE). The American Diabetes Association (ADA) has developed criteria to classify high-risk patients as being anyone that (I) is over 45 years of age, (II) has a family history of T2DM, (III) has a $\mathrm{BMI} \geq 26 \mathrm{~kg} / \mathrm{m}^{2},(\mathrm{IV})$ is sedentary, (V) has hypertension, (VI) has hyperlipidaemia, (VII) is of a certain racial or ethnic group (African American or Hispanic), or (VIII) has had gestational diabetes. The more risk factors an individual has, the higher their risk of developing diabetes. Many studies have applied these criteria prior to undertaking a second level of assessment such as FSB/GCB collection. Similarly, the National Institute for Clinical Excellence (NICE) guidance exists in the UK [47], which outlines similar criteria for high-risk individuals and was used as a "pre-screen" in many of the UK-based studies.

Once a method of study had been determined (Questionnaire, FSB, GCB collection, urinalysis), there are still a number of variables that differentiate the protocols of studies analysed in this review. Accepted "pre-screen" methods include NICE guidance, ADA criteria and validated risk-assessment tools such as "FINDRISC". For a chairside test following "pre-screen", either GCB or FSB collection was employed. Research teams also varied in their biomarker of choice; most chose to measure HbA1c though some opted for 
random blood glucose tests. No clinical trials exist comparing the clinical effectiveness and cost-effectiveness of the various combinations of these methods. Thus, it is difficult to compare, contrast and ultimately decide which protocol offers the greatest diagnostic accuracy.

\section{Which Risk Assessment Protocol Appears Most Robust}

If risk assessment is to be undertaken in dental practices, it should be targeted to individuals at high risk in order to be time and cost-effective [2, 48, 49]. Existing evidence demonstrates that population-based screening is ineffective in terms of cost and clinical outcome [45]. Therefore, in order to determine those who may be at high risk, a targeted approach is required.

Local guidelines and geographical location have an influence on criteria adoption. For example, the ADA criteria are widely accepted and used across the USA and have been employed in many of the studies analysed in this review. NICE guidance suggests that a validated risk assessment tool such as the "Diabetes UK Risk Score" should be used to identify individuals at high risk of T2DM. It advocates that all non-pregnant adults $>40$ years of age, members of highrisk minority ethnic groups (including South Asian, Chinese, African-Caribbean, black African) aged 25-39 years and any people with conditions that place them at increased risk of T2DM should be risk assessed using such validated tools. Where a score above the threshold is obtained, identifying an individual as high risk, they should then undergo a blood test such as HbA1c or fasting plasma glucose [47].

Once a "pre-screen" method has been undertaken to identify those individuals who may be appropriate for formal risk assessment, an appropriate screening method must be selected. Currently, the WHO suggests that provided the patient has symptoms such as polyuria or polydipsia, diagnosis of T2DM can be based on the following:

- A random venous plasma glucose concentration $\geq$ $11.1 \mathrm{mmol} / \mathrm{l}$

- A fasting plasma glucose concentration $\geq 7.0 \mathrm{mmol} / \mathrm{l}$ (whole blood $\geq 6.1 \mathrm{mmol} / \mathrm{l}$ )

- A plasma glucose concentration $\geq 11.1 \mathrm{mmol} / \mathrm{l}, 2 \mathrm{~h}$ after oral administration of $75 \mathrm{~g}$ anhydrous glucose in an oral glucose tolerance test (OGTT).

Where the patient is asymptomatic, a diagnosis should not be based on a single glucose determination, but a further confirmatory plasma venous blood determination would be required. At least one additional glucose test result on another day with a value in the diabetes range is essential, either fasting, from a random sample or from the 2-h post glucose load. If the fasting random values are not diagnostic, the 2-h value should be used. In 2011 the WHO also determined $\mathrm{HbAlc}$ as a suitable method for diagnosing T2DM. They recommended an $\mathrm{HbAlc}$ of $48 \mathrm{mmol} / \mathrm{mol}(6.5 \%)$ as the cutoff point for diagnosing diabetes. A value of less than $48 \mathrm{mmol} / \mathrm{mol}(6.5 \%)$ does not exclude diabetes diagnosed when using glucose tolerance tests and that FSB HbA1c should not be used for diagnosis. Where FSB tests are used, they must be confirmed by laboratory-based venous HbA1c test in all patients [1].

In dental settings, the aim is not to diagnose and manage T2DM but to identify those individuals at high-risk who may benefit from formal intervention and referral to a physician for suitable follow-up. The test used must meet these objectives. Given the potential opportunistic nature of screening in this setting, OGTT and fasting plasma glucose samples are unlikely to be practical or feasible, leaving $\mathrm{HbA} 1 \mathrm{c}$ and random plasma glucose as the remaining viable options.

In 59\% of the studies contributing to this review where some form of risk assessment was undertaken in a dental setting, $\mathrm{HbA1c}$ was selected as the marker of choice. This was likely chosen because HbAlc provides a measure of the glucose bound to haemoglobin and is a reflection of the patient's glucose control over approximately 90 days. HbAlc has recently been advocated by the ADA as the "gold standard" test for screening and diagnosing diabetes, and it does not require the patient to fast prior to their appointment, making it practical for use in a dental setting. The decision to be made then is how to collect the blood sample. A venous sample collected and sent to a laboratory, although considered the gold standard, is timeconsuming and costly and potentially requires certain members of the dental team to undergo additional training. Furthermore, as evidenced in the literature reporting on patient opinions, those tests which provided immediate results were most favoured [33]. Thus, given that chairside HbAlc testing has been shown to consistently provide results that strongly correlate with laboratory assays, and can yield results in as little as $5 \mathrm{~min}$, this may be the preferred option [50].

The two methods of chairside testing covered in the literature are GCB and FSB collection. Six studies compared GCB with FSB; the concordance of the two methods was generally found to be acceptable [51-54]. Some studies reported that patients may find oral sampling less invasive and more comfortable than FSB collection [38], although it was noted that it is not always feasible to obtain blood from the gingival crevice [52-55].

\section{Areas for Further Research}

Though there is an abundance of literature relating to the use of dental practices for identifying individuals with $\mathrm{NDH} /$ T2DM, significant further research is required. Specifically, research into determining the most clinically and costeffective methods of risk assessment, with a suitable followup period to determine the proportion of patients who go on to 
receive a formal diagnosis of NDH/T2DM with a primary care medical practitioner.

\section{Conclusions}

The literature demonstrates that stakeholder opinions, including those of patients, dentists, dental hygienists, physicians and dental students, are all generally positive about the utility of dental settings to identify individuals who are at high risk of developing T2DM or who may unknowingly have the condition. This support crosses healthcare boundaries and has been explored globally. The primary barriers continue to be related to time and cost, though concern about poor follow-up of those individuals highlighted as potentially at-risk was also noted. The literature is replete with a wide range of available methodologies for identifying at-risk individuals. It would appear that a two-staged risk assessment process utilizing a prescreen or validated risk score followed by a chairside $\mathrm{HbAlc}$ sample may be most appropriate approach. However, this has yet to be confirmed by any large-scale clinical trial comparing different combinations of the myriad of screening methodologies available. In summary, although some research has been undertaken to determine opinions and feasibility of utilizing the dental workforce to risk assess for NDH/ T2DM, further work is needed to assess both the clinical and costeffectiveness of such an approach and to establish clear protocols and patient care pathways.

Funding Information Thomas Dietrich reports personal fees from Oxtex Ltd., personal fees from Orthocell, grants from Pfizer, grants from Dentsply, personal fees from Colgate, personal fees from Laramie, and grants from Menarini Richerche, outside the submitted work

\section{Compliance with Ethical Standards}

Conflict of Interest Zehra Yonel, Joanna Batt, Rosemarie Jane E Cerullo, Laura J Grey, and Iain Chapple declare that they have no conflict of interests.

Human and Animal Rights and Informed Consent This article does not contain any studies with human or animal subjects performed by any of the authors.

Open Access This article is licensed under a Creative Commons Attribution 4.0 International License, which permits use, sharing, adaptation, distribution and reproduction in any medium or format, as long as you give appropriate credit to the original author(s) and the source, provide a link to the Creative Commons licence, and indicate if changes were made. The images or other third party material in this article are included in the article's Creative Commons licence, unless indicated otherwise in a credit line to the material. If material is not included in the article's Creative Commons licence and your intended use is not permitted by statutory regulation or exceeds the permitted use, you will need to obtain permission directly from the copyright holder. To view a copy of this licence, visit http://creativecommons.org/licenses/by/4.0/.

\section{References}

Papers of particular interest, published recently, have been highlighted as:

- Of importance

- Of major importance

1. WHO. Use of Glycated Haemoglobin (HbA1c) in the Diagnosis of DiabetesMellitus: WHO Press; 2011. Contract No.: WHO/NMH/ $\mathrm{CHP} / \mathrm{CPM} / 11.1$

2. PHE. NHS Diabetes Prevention Programme (NHS DPP) Nondiabetic hyperglycaemia. London: 2015.

3. Mariano S, Antonio C, Martin B, Iain C. T. DR, Filippo G, et al. scientific evidence on the links between periodontal diseases and diabetes: consensus report and guidelines of the joint workshop on periodontal diseases and diabetes by the international diabetes federation and the European federation of periodontology. J Clin Periodontol. 2018;45(2):138-49.

4. Spencer James CJLM, Vos T, et al. GBD 2017 Disease and Injury Incidence and Prevalence Collaborators. Global, regional, and national incidence, prevalence, and years lived with disability for 354 diseases and injuries for 195 countries and territories, 1990-2017: a systematic analysis for the Global Burden of Disease Study 2017. Lancet. 392: $1789-8588$ Nov 2018. This reference is considered important as it highlights the full impact of Oral and Dental disease and how oral and dental diseases should not be overlooked given the significant burden in terms of Years Lost to Disability.

5. Kassebaum NJ, BernabeE, Dahiya M, Bhandari B, Murray CJ, Marcenes W. Global burden of severe periodontitis in 1990-2010: a systematic review and meta-regression. J Dent Res. 2014;93(11):1045-53.

6. Chapple IL, Genco R, et al. J Periodontol. 2013;84(4 Suppl):S10612 This reference is considered important as it was a landmark manuscript synthesising the evidence relating to Periodontitis and systemic disease.

7. Izuora K, Ezeanolue E, Schlauch K, Neubauer M, Gewelber C, Umpierrez G. Impact of periodontal disease on outcomes in diabetes. Contemp Clin Trials. 2015;41:93-9.

8. Garcia D, Tarima S, Okunseri C. Periodontitis and glycemic control in diabetes: NHANES 2009 to 2012. J Periodontol. 2015;86(4): 499-506.

9. Stanko P, Izakovicova HL. Bidirectional association between diabetes mellitus and inflammatory periodontal disease. A review. Biomed Pap Med Fac Univ Palacky Olomouc Czech Repub. 2014;158(1):35-8.

10. Sanz M, Ceriello A, Buysschaert M, Chapple I, Demmer RT, Graziani F, et al. Scientific evidence on the links between periodontal diseases and diabetes: Consensus report and guidelines of the joint workshop on periodontal diseases and diabetes by the International Diabetes Federation and the European Federation of Periodontology. J Clin Periodontol. 2018;45(2):138-49 This reference is important as it builds on the previous consensus report with a particular focus on Periodontitis and Diabetes.

11. Harvey PM. Control of severe periodontitis with reduction of infection and some recovery of clinical attachment in a patient with initially undiagnosed diabetes. J N Z Soc Periodontol. 1986;61:12-7.

12. Position Paper; Diabetes and Periodontal Diseases. J Periodontol. 1999;70(8):935-49.

13. Paper P. Diabetes and periodontal diseases. J Periodontol. 2000;71(4):664-78.

14. Al-Khabbaz AK. Type 2 diabetes mellitus and periodontal disease severity. Oral Health Prev Dent. 2014;12(1):77-82.

15. Artese HP, Foz AM, Rabelo Mde S, Gomes GH, Orlandi M, Suvan J, et al. Periodontal therapy and systemic inflammation in type 2 diabetes mellitus: a meta-analysis. PLoS One. 2015;10(5):e0128344. 
16. Bascones-Martinez A, Gonzalez-Febles J, Sanz-Esporrin J. Diabetes and periodontal disease. Review of the literature. Am J Dent. 2014;27(2):63-7.

17. Chapple IL, Borgnakke WS, Genco RJ. Hemoglobin A1c levels among patients with diabetes receiving nonsurgical periodontal treatment. JAMA. 2014;311(18):1919-20.

18. Deschner J, Haak T, Jepsen S, Kocher T, Mehnert H, Meyle J, et al. Diabetes mellitus and periodontitis. Bidirectional relationship and clinical implications. A consensus document. Internist. 2011;52(4):466-77.

19. Bascones-Martinez A, Munoz-Corcuera M, Bascones-Ilundain J. Diabetes and periodontitis: a bidirectional relationship. Med Clin. 2015;145(1):31-5.

20. Casanova L, Hughes FJ, Preshaw PM. Diabetes and periodontal disease: a two-way relationship. Br Dent J. 2014;217(8):433-7.

21. Mealey B. Diabetes and periodontal diseases. J Periodontol. 1999;70(8):935-49.

22. Mirza W, Saleem MS, Patel G, Chacko P, Reddy S, Schaefer R, et al. Early screening strategies for diabetes mellitus by leveraging dental visits using optimal screening tools available onsite. Cureus. 2018;10(11):e3641.

23. Taylor GW, Borgnakke WS. Periodontal disease: associations with diabetes, glycemic control and complications. Oral Dis. 2008;14(3): 191-203.

24. D'Aiuto F, Gkranias N, Bhowruth D, Khan T, Orlandi M, Suvan J, et al. Systemic effects of periodontitis treatment in patients with type 2 diabetes: a 12 month, single-centre, investigator-masked, randomised trial. Lancet Diabetes Endocrinol. 2018;6(12):954-65.

25. Tonetti MS, Jepsen S, Jin L, Otomo-Corgel J. Impact of the global burden of periodontal diseases on health, nutrition and wellbeing of mankind: a call for global action. J Clin Periodontol. 2017;44(5):456-62.

26. Listl S, Galloway J, Mossey PA, Marcenes W. Global economic impact of dental diseases. J Dent Res. 2015;94(10):1355-61.

27. Moher D, Shamseer L, Clarke M, Ghersi D, Liberati A, Petticrew M, et al. Preferred reporting items for systematic review and meta-analysis protocols (PRISMA-P) 2015 statement. Syst Rev. 2015;4(1):1.

28. Anders PL, Davis EL, McCall WD Jr. Dental students' glucometer experience and attitudes toward diabetes counseling, monitoring, and screening: a comparative study. J Dent Educ. 2014;78(9):1263-7.

29. Esmeili T, Ellison J, Walsh MM. Dentists' attitudes and practices related to diabetes in the dental setting. J Public Health Dent. 70(2): 108-14.

30. Friman G, Hultin M, Nilsson GH, Wardh I. Medical screening in dental settings: a qualitative study of the views of authorities and organizations. BMC Res Notes. 2015;8:580.

31. Greenberg BL, Glick M, Frantsve-Hawley J, Kantor ML. Dentists' attitudes toward chairside screening for medical conditions. J Am Dent Assoc. 2010;141(1):52-62.

32. Greenberg BL, Kantor ML, Bednarsh H. American dental hygienists' attitudes towards chairside medical screening in a dental setting. Int J Dent Hyg. 2017;15(4):e61-e8.

33. Greenberg BL, Kantor ML, Jiang SS, Glick M. Patients' attitudes toward screening for medical conditions in a dental setting. J Public Health Dent. 2012;72(1):28-35.

34. Greenberg BL, Thomas PA, Glick M, Kantor ML. Physicians' attitudes toward medical screening in a dental setting. J Public Health Dent. 2015;11.

35. Greenblatt AP, Estrada I, Schrimshaw EW, Metcalf SS, Kunzel C, Northridge ME. Acceptability of Chairside Screening for Racial/ Ethnic Minority Older Adults: A Qualitative Study. JDR Clin Transl Res. 2017;2(4):343-52.

36. Sansare K, Raghav M, Kasbe A, Karjodkar F, Sharma N, Gupta A, et al. Indian patients' attitudes towards chairside screening in a dental setting for medical conditions. Int Dent J. 2015;65(5):269-76.

37. Scambler S, Asimakopoulou K. Summary of: Patients' attitudes towards screening for diabetes and other medical conditions in the dental setting. Br Dent J. 2014;216(1):34-5.
38. Rosedale MT, Strauss SM. Diabetes screening at the periodontal visit: patient and provider experiences with two screening approaches. Int J Dent Hyg. 2012;10(4):250-8.

39. Creanor S, Millward BA, Demaine A, Price L, Smith W, Brown N, et al. Patients' attitudes towards screening for diabetes and other medical conditions in the dental setting. Br Dent J. 2014;216(1):E2.

40. Doherty R. Summary of: Patients' attitudes towards screening for diabetes and other medical conditions in the dental setting. Br Dent J. 2014;216(1):34-5.

41. Yonel Z, Sharma P, Yahyouche A, Jalal Z, Dietrich T, Chapple IL. Patients' attendance patterns to different healthcare settings and perceptions of stakeholders regarding screening for chronic, noncommunicable diseases in high street dental practices and community pharmacy: a cross-sectional study. BMJ Open. 2018;8(11).

42. Greenberg BL, Kantor ML, Bednarsh H. American dental hygienists' attitudes towards chairside medical screening in a dental setting. Int J Dent Hyg. 2016;28:28.

43. Bossart M, Calley KH, Gurenlian JR, Mason B, Ferguson RE, Peterson T. A pilot study of an $\mathrm{HbAlc}$ chairside screening protocol for diabetes in patients with chronic periodontitis: the dental hygienist's role. Int J Dent Hyg. 2015;23.

44. Bould K, Scott SE, Dunne S, Asimakopoulou K. Uptake of screening for type 2 diabetes risk in general dental practice; an exploratory study. Bdj. 2017;222:293

45. Engstrom S, Berne C, Gahnberg L, Svardsudd K. Effectiveness of screening for diabetes mellitus in dental health care. Diabet Med. 2013;30(2):239-45.

46. Barasch A, Gilbert GH, Spurlock N, Funkhouser E, Persson L-L, Safford MM, et al. Random plasma glucose values measured in community dental practices: findings from the dental practicebased research network. Texas Dent J. 2013;130(4):291-7.

47. NICE. Type 2 diabetes: prevention in people at high risk. NiCE: National Institute for Health and Care Excellence, updated 2017.

48. van den Donk M, Sandbaek A, Borch-Johnsen K, Lauritzen T, Simmons RK, Wareham NJ, et al. Screening for type 2 diabetes. Lessons from the ADDITION-Europe study. Diabet Med. 2011;28(11):1416-24.

49. Penn L, Rodrigues A, Haste A, Marques MM, Budig K, Sainsbury $\mathrm{K}$, et al. NHS diabetes prevention Programme in England: formative evaluation of the programme in early phase implementation. BMJ Open. 2018;8(2):e019467.

50. Arrendale JR, Cherian SE, Zineh I, Chirico MJ, Taylor JR. Assessment of glycated hemoglobin using A1CNow+ point-ofcare device as compared to central laboratory testing. J Diabetes Sci Technol. 2008;2(5):822-7.

51. Ardakani MR, Moeintaghavi A, Haerian A, Ardakani MA, Hashemzadeh M. Correlation between levels of sulcular and capillary blood glucose. J Contemp Dent Pract. 2009;10(2):10-7.

52. Khader YS, Al-Zu'bi BN, Judeh A, Rayyan M. Screening for type 2 diabetes mellitus using gingival crevicular blood. Int J Dent Hyg. 2006;4(4):179-82.

53. Rajesh KS, Irshana R, Kumar MSA, Hegde S. Effectiveness of glucometer in screening diabetes mellitus using gingival crevicular blood. Contemp Clin Dent. 2016;7(2):182-5.

54. Sibyl S, Bennadi D, Kshetrimayum N, Manjunath M. Correlations between gingival crevicular blood glucose and capillary blood glucose: a preliminary report. J Lab Physicians. 2017;9(4):260-3.

55. Muller HP, Behbehani E. Screening of elevated glucose levels in gingival crevice blood using a novel, sensitive self-monitoring device. Med Princ Pract. 2004;13(6):361-5.

Publisher's Note Springer Nature remains neutral with regard to jurisdictional claims in published maps and institutional affiliations. 